\title{
Spin-polarized tunneling between an antiferromagnet and a ferromagnet: First-principles calculations and transport theory
}

\author{
J. Enkovaara, D. Wortmann, and S. Blügel \\ Institut für Festkörperforschung, Forschungszentrum Jülich, Germany \\ (Received 13 April 2006; revised manuscript received 12 April 2007; published 21 August 2007)
}

\begin{abstract}
By combining first-principles calculations with transport theory we investigate the origin of the magnetoresistance of a magnetic tunnel junction consisting of a ferromagnetic and an antiferromagnetic lead. The (001) oriented $\mathrm{Fe} / \mathrm{vacuum} / \mathrm{Cr}$ planar junction serves as model junction. Even though the conduction electrons of antiferromagnetic $\mathrm{Cr}$ are spin-degenerate, it is possible to observe magnetoresistance due to two mechanisms: Firstly, the surface magnetism of $\mathrm{Cr}$ creates a spin-dependent potential barrier, and secondly, exchange-split surface states and resonances result in a tunneling conductance which depends on the relative orientation of the $\mathrm{Fe}$ and $\mathrm{Cr}$ magnetizations. Spin-dependent tunneling between a ferromagnet and an antiferromagnet happens frequently in tunneling setups such as in spin-polarized scanning tunneling microscopy or magnetic tunnel junctions for magnetic random access memory.
\end{abstract}

DOI: 10.1103/PhysRevB.76.054437

PACS number(s): 71.15.Ap, 73.40.-c, 72.10.-d, 73.20.At

\section{INTRODUCTION}

The effect of spin-polarized tunneling of electrons through vacuum or an insulating barrier has recently attracted increased attention due to experimental progress in two fields: (i) The spin-polarized tunneling microscopy ${ }^{1}$ (SP-STM) which has developed into an important engine driving the field of nanomagnetism and (ii) the fabrication of planar tunnel junctions showing huge tunneling magnetoresistance effect of over $400 \%$ at room temperature. ${ }^{2-4}$

The difference in the conductance due to a change in the relative orientation of the magnetization directions of tip and sample or of the two magnetic leads in the case of a planar tunnel barrier, is frequently related to the spin-polarization of the conducting electrons. The simplest model proposing such a relation is due to Julliere ${ }^{5}$ and derives the magnetoresistance (MR), i.e., the relative difference of the resistances in the parallel $R_{P}$ and antiparallel $R_{\mathrm{AP}}$ alignments of the magnetization, from the density of states at the Fermi level $n_{\uparrow / \downarrow}^{L / R}$ for the two spin directions $\uparrow / \downarrow$ and the two leads $L / R$

$$
\mathrm{MR}=\frac{R_{\mathrm{AP}}-R_{P}}{R_{P}}=\frac{n_{\uparrow}^{L} n_{\uparrow}^{R}+n_{\downarrow}^{L} n_{\downarrow}^{R}}{n_{\uparrow}^{L} n_{\downarrow}^{R}+n_{\downarrow}^{L} n_{\uparrow}^{R}}-1 .
$$

While this simple model can provide some insight into the basic mechanism of spin-polarized tunneling with ferromagnetic leads, it cannot easily be applied to tunneling between an ferromagnet and an antiferromagnet. The density of states of the antiferromagnet is spin-degenerate $n_{\uparrow}=n_{\downarrow}$ and consequently, Julliere's model [Eq. (1)] predicts no magnetoresistance in such setups. In contrast to this finding, one of the most successful realizations of SP-STM uses an antiferromagnetic Cr film tip. ${ }^{6}$ These experiments have been motivated by the need to minimize the magnetic (e.g., dipole) interaction between tip and sample changing or inflicting with the magnetization of the sample. As $\mathrm{Cr}$ is an antiferromagnet, consisting of ferromagnetic (001) planes coupling antiferromagnetically from plane to plane along the plane normal (with a some small deviation off the lattice matched value of $\pi / a$ due to a temperature dependent incommensu- rability of $\delta=0.952 \times \pi / a$, where $a$ is the bulk Cr lattice constant), the magnetic dipole interaction between tip and sample is zero and the magnetic interaction is of higher order and thus tiny and in general negligible. Analogously to these SP-STM experiments showing a magnetic contrast with an antiferromagnetic tip, a large MR value has also be found in planar tunnel junctions where one electrode contained an antiferromagnetic spacer. $^{7}$

Different effects have been proposed to explain the origin of the magnetic signal in these experiments including interface effects and a spin-filtering effect due to a symmetry mismatch of the states in planar junctions. In this paper, we aim at shining more light onto the issue by investigating the spin-dependent tunneling between an antiferromagnet and a ferromagnet. We calculate the actual tunneling conductance between semi-infinite $\mathrm{Cr}(001)$ and $\mathrm{Fe}(001)$ surfaces separated by a vacuum barrier as a simple model for a planar tunnel junction as well as for SP-STM with a blunt tip. The major question we will answer is whether a tunnel junction between a ferromagnet and an antiferromagnet exhibits a conductance which depends on the relative alignment between the magnetic moments of the ferro- and antiferromagnet. The relative alignment between the moments of the ferro- and antiferromagnetic lead is defined throughout this paper by the alignment of their respective surface moments. We show that there are two mechanisms which give rise to a nonzero magnetoresistance: (i) The surface magnetism of $\mathrm{Cr}$ leads to a spin-dependent potential barrier and (ii) the presence of exchange-split surface states and resonances.

\section{DETAILS OF CALCULATIONS}

Our calculations are carried out within the density functional theory (DFT) using the local spin-density approximation (LSDA) for the exchange-correlation potential. ${ }^{8}$ We use the full-potential linearized augmented plane wave (FLAPW) method as implemented in the FLEUR code. ${ }^{9}$ The model system consists of semi-infinite $\mathrm{Cr}(001)$ separated from semi-infinite $\mathrm{Fe}(001)$ by a vacuum barrier equivalent to the thickness of five atomic layers (13.48 a.u.). The same 
lattice constant of 5.39 a.u. is used throughout the whole structure, which is consistent with the theoretical lattice constants of $\mathrm{Cr}$ and $\mathrm{Fe}$ and in good agreement with experimental values of 5.44 a.u. and 5.42 a.u. for bulk $\mathrm{Cr}$ and $\mathrm{Fe}$, respectively. The magnetic structure of $\mathrm{Cr}$ is layerwise antiferromagnetic, neglecting the small incommensurability. ${ }^{10}$ The potential of the $\mathrm{Fe} / \mathrm{vacuum} / \mathrm{Cr}$ system is calculated from a super-cell. Basis functions are included up to a plane wave cutoff $k_{\max }=3.8$ a.u. $^{-1}$ resulting in approximately 100 augmented plane waves per atom. Then, the boundary conditions corresponding to the semi-infinite $\mathrm{Fe}$ and $\mathrm{Cr}$ are treated with the embedded Green function method ${ }^{11}$ which has been recently implemented within the FLAPW-method. ${ }^{12,13}$

\section{THEORY}

The standard approach to the calculation of the conductance of coherently tunneling electrons between the $\mathrm{Fe}(001)$ and $\mathrm{Cr}(001)$ leads is the Landauer formula. ${ }^{14}$ Both $\mathrm{Fe}(001)$ and $\mathrm{Cr}(001)$ surfaces have surface states ${ }^{15}$ who contribute to the conductance in the experimental setups. However, in a single-particle picture of coherent tunneling such as the Landauer formula, localized surface states do not carry current as they are orthogonal to the delocalized states. The tunneling from localized states is due to inelastic processes and disorder, but for weakly coupled electrodes in the limit of small tunneling current it is possible to approximate these effects with Bardeen tunneling Hamiltonian formalism. ${ }^{16}$ For more detailed discussion of the differences between the Landauer and Bardeen description of tunneling and the formulation of the tunneling current in the Bardeen model within the framework of the embedding method, we refer the reader to Ref. 17.

The current density in the Bardeen formulation $j^{B}$ can be written in terms of the transmission probabilities $\left|t_{i j}^{B}\right|^{2}$ as

$$
j^{B}=\frac{h}{e^{2}} \sum_{i j}\left[f^{l}\left(E_{i}\right)-f^{r}\left(E_{j}+e V\right)\right] \delta\left(E_{i}-E_{j}-e V\right)\left|t_{i j}^{B}\right|^{2},
$$

where $V$ is the bias voltage, $f^{l, r}(E)$ are the Fermi-distribution functions of the left and the right leads, and the summation is over all the states in the left lead $(i)$ and in the right lead $(j)$. In the zero bias limit one obtains the linear response conductance $\Gamma^{B}$

$$
\Gamma^{B}=\frac{h}{e^{2}} T\left(E_{F}\right),
$$

where the total transmission probability $T=\Sigma_{i j}\left|t_{i j}^{B}\right|^{2}$ is now evaluated at the Fermi level, $E_{F}$. Although this expression looks identical to the well-known Landauer formula, there is one important difference: In the Bardeen approach the localized surface states are included in the summation over the incoming $(i)$ and the outgoing $(j)$ channels, while the Landauer formula includes only the bulk-like states. For these bulk-like states, the transmission probabilities $\left|t_{i j}^{B}\right|^{2}$ approach the values calculated by the Landauer formula, in particular for high tunneling barriers, ${ }^{17}$ which is the case for the vacuum barriers discussed in this paper. When one electrode in the Bardeen formulation is modeled by a single atom with an $s$-wave and constant density of states, one obtains the Tersoff-Hamann model.

When the electrodes are magnetic, the transmission probability depends naturally on the spin as well as on the relative orientation of the magnetizations of the electrodes. This gives rise to magnetoconductance (or equivalently magnetoresistance), which is at the heart of the devices using magnetic tunnel junctions and gives the magnetic contrast in SPSTM. In SP-STM, one can measure the change in the conductance by rotating the magnetization of either the sample or the tip. Here, as in the most experiments, we consider only two magnetic configurations where the magnetizations of the two electrodes (the sample and the tip in SPSTM) are either parallel $(P)$ or antiparallel (AP). The magnetoresistance (MR) is usually expressed in terms of the relative change in the resistance

$$
\mathrm{MR}=\frac{R_{\mathrm{AP}}-R_{P}}{R_{P}} .
$$

In addition to the transmission probability at the Fermi level, which is directly proportional to the linear response conductance, we look also at the energy dependence of the transmission probability. Even though it is not directly related to the finite bias conductance, the energy dependence gives some information about the tunneling characteristics at an applied bias voltage.

\section{RESULTS AND DISCUSSION}

At first, we look into the case where the incident electrons travel normal to the surface, i.e. we discuss electrons from the center $\left(\vec{k}_{\|}=0\right.$ or $\bar{\Gamma}$-point $)$ of the two-dimensional Brillouin zone. In Fig. 1 we show the bulk band structures of Fe and $\mathrm{Cr}$ along those high-symmetry lines which project into the $\bar{\Gamma}$-point. In addition, the energy positions of the surface states are indicated. For Fe one obtains a spin-split band structures of majority and minority states. The bulk and surface magnetic moments are $2.25 \mu_{B}$ and $2.85 \mu_{B}$, respectively, in good agreement with previous studies. ${ }^{18,19}$ The exchangesplit surface states appear at $-1.8 \mathrm{eV}(0.2 \mathrm{eV})$ for majority (minority) spin, agreeing also with previous experiments and theory ${ }^{15}$ As bulk $\mathrm{Cr}$ is antiferromagnetic with a lattice periodicity normal to the surface (about) twice as large as that of ferromagnetic $\mathrm{Fe}$, the bulk electronic structure of $\mathrm{Cr}$ is spindegenerate and the states along $\Gamma-X$ are related to the states between $\Gamma-H$ of $\mathrm{Fe}$ by band-folding. Although, the net magnet moment of bulk $\mathrm{Cr}$ is zero, the $\mathrm{Cr}(001)$ surface is known to be magnetic with an enhanced surface magnetic moment. ${ }^{20,21}$ In our calculations, we obtain a bulk and surface magnetic moment of $0.75 \mu_{B}$ and $2.20 \mu_{B}$, which compare well with other theoretical results. ${ }^{15,22,23}$ Because of the surface magnetism, the surface states are exchange split and appear at $-1.20 \mathrm{eV}(0.43 \mathrm{eV})$ for majority (minority) spin. However, these energies are sensitive to the structural details and the surface magnetic moment. Different experiments have reported different binding energies for the surface states, but in general a minority spin surface state is found near the Fermi level either below ${ }^{15}$ or above. ${ }^{24}$ 

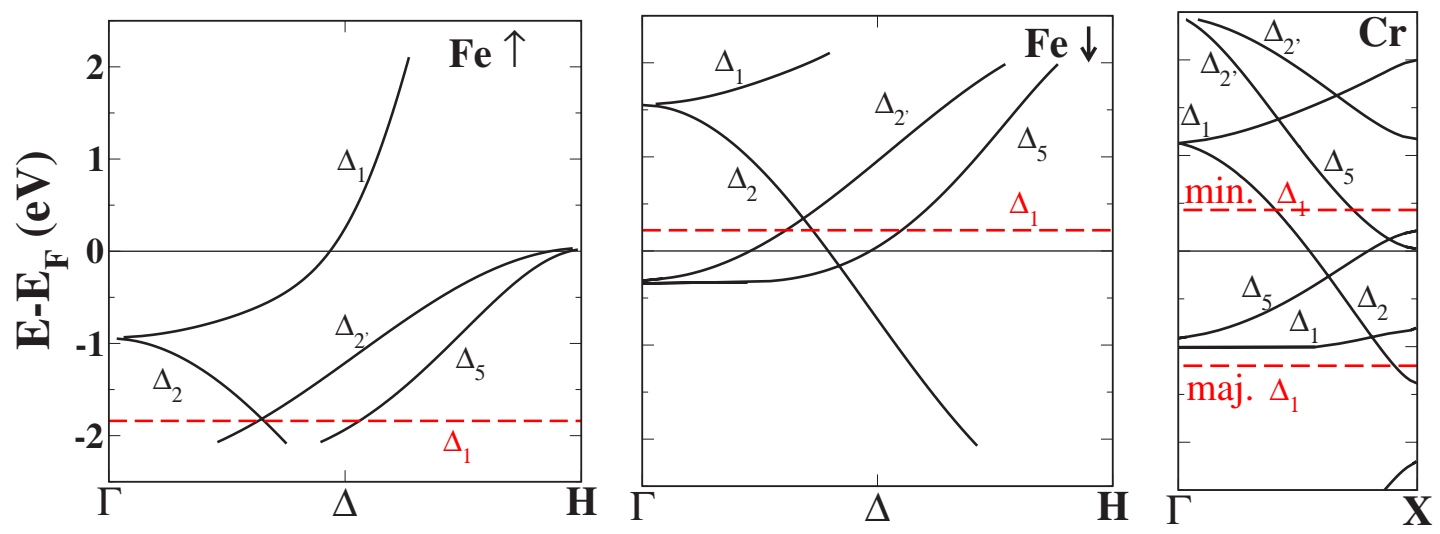

FIG. 1. (Color online) Bulk band structures projecting onto $\bar{\Gamma}$ point. The positions of the surface states are indicated by the horizontal dashed lines. The left panel shows the Fe majority bands, the central one the Fe minority states and the right panel the Cr bands.

As elucidated in previous works ${ }^{25-27}$ the symmetry of the electronic states plays an important role in tunneling. First, only those electronic states having the same symmetry on both sides of the barrier can couple. Secondly, the symmetry affects also the decay of the electronic wave function into the vacuum. In general, the less nodes or kinetic energy, respectively, the wave function has in the plane perpendicular to the decay direction, the slower is the decay. This means that within the two-dimensional symmetry group of the $\mathrm{Fe}(001)$ and $\operatorname{Cr}(001)$ surfaces, states having $\Delta_{1}$ symmetry decay slowest into the vacuum. The $\Delta_{1}$ bands start at the $\bar{\Gamma}$ point at binding energies around $-1 \mathrm{eV}(1.5) \mathrm{eV}$ for $\mathrm{Fe}$ majority (minority) states, and $1.1 \mathrm{eV}$ for $\mathrm{Cr}$, and they are dispersing upwards in energy. Additionally, the surface states marked in Fig. 1 are of $\Delta_{1}$ symmetry. At metal surfaces SP-STM experiments can be carried out with bias voltages up to $\sim \pm 1 \mathrm{~V}$. In this energy range the only bulklike $\Delta_{1}$-electrons originate from the Fe majority band. The minority $\Delta_{1}$ surface states of both $\mathrm{Fe}$ and $\mathrm{Cr}$ may give additional contributions in this particular energy range.

The spin-resolved transmission probabilities $T_{\uparrow(\downarrow)}^{\mathrm{P}(\mathrm{AP})}$ for majority ( $\uparrow)$ and minority $(\downarrow)$ states at the $\bar{\Gamma}$ point as function of the energy are shown in Fig. 2 for both magnetic configurations, where the magnetic moments of the outermost $\mathrm{Fe}$ and $\mathrm{Cr}$ layers are aligned either parallel $(P)$ or antiparallel (AP). The terms majority (spin-up) and minority (spin-down) are defined with respect to a global spin-quantization axis of the tunnel junction, which we define here to be parallel to the magnetic moment of the $\mathrm{Fe}$ surface. Hence, in the parallel configuration the $\mathrm{Cr}$ spin-up electrons are those with a larger density of states at the outermost $\mathrm{Cr}(001)$ surface layer while in the antiparallel configuration the spin-down electrons are those with the larger the density of states in that layer.

$\mathrm{Cr}$ exhibits a wide energy region without any $\Delta_{1}$ state and therefore the bulk states with normal incidence will have a small transmission probability for both the parallel and antiparallel magnetic alignments. Only at around $1.1 \mathrm{eV}$ above the Fermi level, where the $\Delta_{1}$ band of the Fe majority states overlaps with the $\mathrm{Cr} \Delta_{1}$ states a significant transmission probability can be found with a strongly increasing transmission at higher energies. For the Fe-minority states the overlap begins higher in energy and the transmission probability starts to increase at around $1.5 \mathrm{eV}$. One can see that for both spin directions the energy at which a significant transmission sets in does not depend on the magnetic configuration. This point is determined only by the bulk band structure which is spin-degenerate for Cr. However, the surface magnetism of $\mathrm{Cr}$ results in a spin dependent potential barrier and therefore the actual magnitude of transmission depends on the relative magnetic alignment of $\mathrm{Cr}$ tip and Fe sample.

In addition, for the antiparallel alignment of tip and sample a narrow peak can be seen in the spin-up channel of the transmission probability arising from the surface states. In principle, the surface states result in a delta-function like peak in the transmission probability. In order to resolve these peaks numerically a small imaginary part $(\sim 1 \mathrm{meV} \sim 12 \mathrm{~K})$ is added in the computation to the energy which finally gives the peak a finite height and width. The Fe surface states do not cross the bulk $\mathrm{Cr} \Delta_{1}$ bands and therefore cannot contribute to the conductance. On the other hand, the $\mathrm{Cr}$ surface state near the Fermi level overlaps with the spin-up $\Delta_{1}$ band
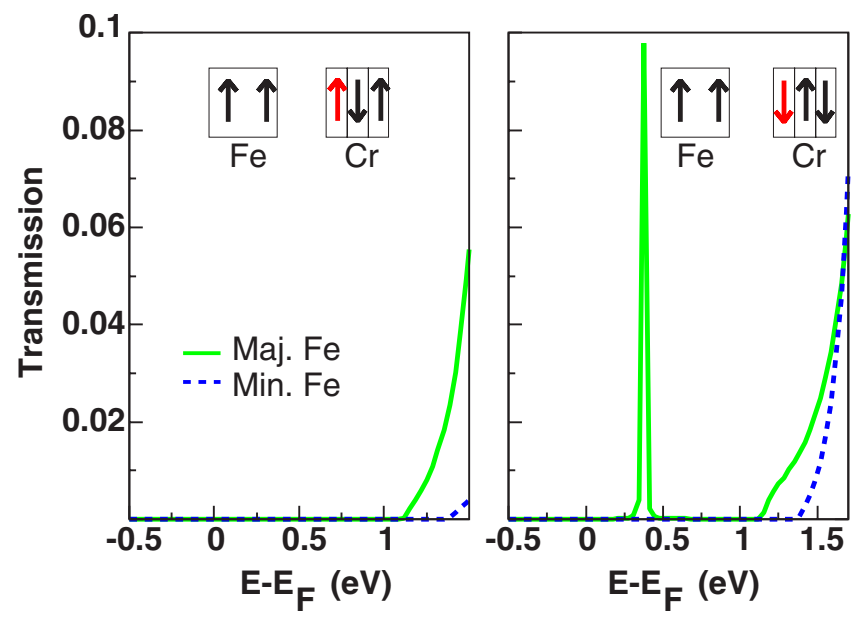

FIG. 2. (Color online) Spin-resolved transmission probabilities at $\bar{\Gamma}$ as a function of the electron energy for parallel $(P)$ (left panel) and antiparallel (AP) (right panel) alignment of tip and sample magnetization. The main solid (dashed) curve shows the majority (minority) spin channel. 

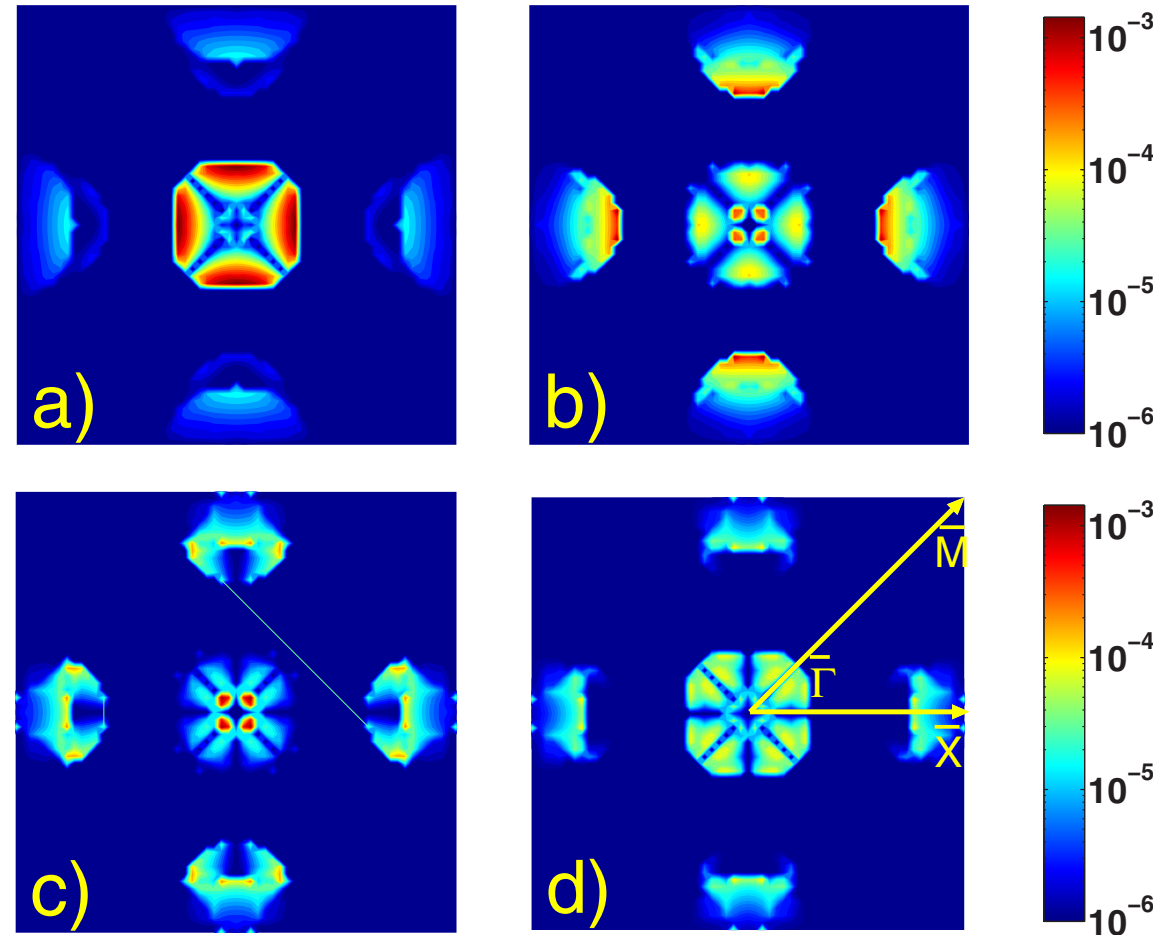

FIG. 3. (Color online) Transmission probability (logarithmic scale) over the two-dimensional Brillouin zone at the Fermi energy. (a) Majority spin-channel, parallel alignment, (b) majority spin-channel, antiparallel alignment, (c) minority spin-channel, parallel alignment, and (d) minority spin-channel, antiparallel alignment.

of Fe which starts at $-1 \mathrm{eV}$ and thus can give a finite contribution to the conductance if the surface moments are properly aligned. When the magnetic alignment is parallel, the $\mathrm{Cr}$ surface state is of spin-down character and cannot tunnel into the bulk $\Delta_{1}$ band of the Fe spin-up states due to spinconservation during the tunneling process. However, in the antiparallel alignment the $\mathrm{Cr}$ surface state has the same spin as the overlapping $\mathrm{Fe}$ band, producing sharp feature in the tunneling conductance. Even though the positions of the $\mathrm{Cr}$ surface states are delicate, some conclusions can be drawn. As long as there is a Cr surface state near the Fermi level, a large change in the tunneling conductance, which is a Fermilevel property, at $\vec{k}_{\|}=0$ with respect to the magnetic alignment can be expected, as this surface state contributes to the conductance only in the anti-parallel magnetic alignment. Finally, the Fe surface states cannot tunnel into bulk $\mathrm{Cr}$ states and thus they do not contribute to the current.

The above discussion is strictly valid only at the $\bar{\Gamma}$ point as in general $\vec{k}_{\|}$points the symmetry does no longer forbid the tunneling from slowly decaying bulk states. However, close to $\bar{\Gamma}$-point transmission probability from bulk states remains small and there is significant tunneling only in the antiparallel magnetic configuration from the localized $\mathrm{Cr}$ states which are no longer pure surface states but surface resonances.

The transmission probability discussed so far does not include the effect of any bias voltage. Neither the shift in the band alignment induced by the bias voltage nor the modifications of the surface potentials due to screening effects are included. Nevertheless, the peaks in the transmission can give an indication of the basic features to be expected in an experimental $d I / d V$ plot as they provide some insight into the coupling strength of the different states. The transmission probability close to the Fermi level is directly related to the linear response conductance for small bias voltages by means of Eq. (3).

As the transmission probability decays exponentially, $\Gamma$ $\sim \exp \left(-\right.$ const $\left|k_{\|}\right|^{2}$ ), in tunnel junctions of large barriers, normally the electronic states near the $\bar{\Gamma}$-point dominate the transmission. Due to symmetry this contribution is very small at the Fermi level, which necessitates the investigation of all contributions across the Brillouin zone in more detail. In Fig. 3 we show the transmission probability at the Fermi level which gives the linear response conductance when integrated over the two-dimensional Brillouin zone.

All the figures show some very similar features. As the symmetry restrictions just discussed do not allow the Fe $\Delta_{1}$ band to couple to any $\mathrm{Cr}$ state at the $\bar{\Gamma}$ point the transmission is very low. Similar restrictions hold also along some high symmetry directions. In all the cases, the $\langle 11\rangle$ directions show significant transmission only near the center of the Brillouin zone. In the $\langle 10\rangle$ directions, the majority spin transmission [Figs. 3(a) and 3(b)] shows some transmission also in the central region, but expect the narrow ribbon, the minority spin transmission [Fig. 3(c) and 3(d)] is larger only close to the edge of the Brillouin zone. For general $\vec{k}_{\|}$points there are no symmetry restrictions and the $\mathrm{Fe}$ and $\mathrm{Cr}$ bands start to couple by forming coherent superpositions. At the same time, when moving away from the $\bar{\Gamma}$ point, the transmission probability decreases due to the increased exponen- 
tial decay which results finally in the flowerlike transmission shapes.

All the symmetry related features result solely from the bulk-band structures. As the $\mathrm{Cr}$ bulk bands are spindegenerate, the symmetry restrictions are the same for both the parallel and antiparallel magnetic alignment of the leads. Differences between majority and minority spin result from the exchange split Fe bands. Also for the general $\vec{k}_{\|}$points, the transmission is largely determined by the available channels in the bulk $\mathrm{Cr}$, and thus there are similar transmission shapes in both spins and in both magnetic alignments.

Even though the general shapes do not depend on the magnetic configuration, the results show clearly that the actual magnitude of the transmission probability changes substantially when the $\mathrm{Cr}$ magnetization is flipped with respect to the Fe magnetization. Due to the surface magnetism of $\mathrm{Cr}$, the potential barrier at the $\mathrm{Cr}$ interface is spin-dependent causing a large magnetoresistance. Although a precise value depends on all details of a real surface and a fully converged value requires a huge number of $\vec{k}_{\|}$points, our present calculations point to over $100 \%$ magnetoresistance.

The strong dependence of the conductance on the interfacial magnetizations is a general feature present in both planar geometries as a discussed here as well as in the SP-STM setup with a strong structural asymmetry between the two sides. Our result can be interpreted in terms of the simple Tersoff Hamann model ${ }^{28}$ relating the tunneling current to the density of states leaking out into the vacuum barrier. In the magnetic case this model expresses the magnetic signal in the STM, i.e., the magnetoresistance, to the magnetization densities $M_{T / S}$ of the two electrodes [tip $(T)$ and sample $(S)$ in STM] in the vacuum ${ }^{29}$

$$
\operatorname{MR} \propto \vec{M}_{T} \cdot \vec{M}_{S}
$$

For a ferromagnetic sample $M_{S}$ can be related to the bulk magnetization and the decay of the corresponding states into the barrier. In the case of the antiferromagnetic tip, the bulk magnetization vanishes, however, the magnetic moment of the surface layer induces a different barrier for bulk electrons of the two spin directions. Hence the surface magnetization can effectively polarize $M_{T}$ in the vacuum and lead to a significant dependence of the conductance on the magnetic alignment of tip and sample.

\section{CONCLUSIONS}

In this paper we explored the presence of magnetoresistance in a tunnel junction when one of the electrodes is an antiferromagnet. We found, that the conductance depends on the relative alignment between the respective $\mathrm{Fe}$ and $\mathrm{Cr}$ surface moments and identified two essential mechanisms: (i) Bulk electrons, which are spin-degenerate deep in the antiferromagnetic electrode experience a tunneling barrier, which depends on the magnetic alignment of the surface atoms. (ii) Exchange split surface states on the surface of the antiferromagnet have an intrinsic spin dependence and as such they will contribute to the magnetoresistance.

For the $\mathrm{Fe} /$ vacuum/Cr junction the magnetization dependent barrier at the $\mathrm{Cr}$ interface contributes to a TMR ratio which is positive (higher conductance for parallel alignment between $\mathrm{Fe}$ and $\mathrm{Cr}$ surface) with an estimated value larger than $100 \%$. The TMR ratio due to the surface state is negative.

We identified these two contributions as a function of electron energy and $\vec{k}_{\|}$vector of the surface Brillouin zone. We have discussed that in ideal planar junctions the symmetry of the electronic states plays an important role. The presence of a spin-dependent potential barrier resulting from the surface magnetism of $\mathrm{Cr}$ does not depend on the details of our idealized model of planar junctions. Thus our conclusions apply to SP-STM setups as well as to the realistic tunnel junctions such as $\mathrm{Fe} / \mathrm{MgO} / \mathrm{Cr}$. On the other hand, due to the uncertainty of the tip geometry and its effect on the surface state, in practice the contribution of such states to spindependent tunneling is very hard to predict. Our results seem to indicate that it can be even advantageous to work with rather blunt $\mathrm{Cr}$ tips exploiting the strong spin-dependence and the spatial resolution of the $d_{z}^{2}$ surface state of $\mathrm{Cr}(001)$ for spin-resolving imaging and spectroscopy.

\section{ACKNOWLEDGMENTS}

J.E. has been supported by the EU Marie Curie IntraEuropean Fellowship.
${ }^{1}$ M. Bode, Rep. Prog. Phys. 66, 523 (2003).

${ }^{2}$ S. S. P. Parkin, C. Kaiser, A. Panchula, P. M. Rice, B. Hughes, M. Samant, and S.-H. Yang, Nat. Mater. 3, 862 (2004).

${ }^{3}$ S. Yuasa, T. Nagahama, A. Fukushima, Y. Suzuku, and K. Ando, Nat. Mater. 3, 868 (2004).

${ }^{4}$ S. Yuasa, A. Fukushima, H. Kubota, Y. Suzuki, and K. Ando, Appl. Phys. Lett. 89, 042505 (2006), URL http://link.aip.org/ link/?APL/89/042505/1.

${ }^{5}$ M. Julliere, Phys. Lett. 54A, 225 (1975).

${ }^{6}$ A. Kubetzka, M. Bode, O. Pietzsch, and R. Wiesendanger, Phys. Rev. Lett. 88, 057201 (2002).

${ }^{7}$ T. Nagahama, S. Yuasa, E. Tamura, and Y. Suzuki, Phys. Rev.
Lett. 95, 086602 (2005), URL http://link.aps.org/abstract/PRL/ v95/e086602.

${ }^{8}$ V. L. Moruzzi, J. F. Janak, and A. R. Williams, Calculated Electronic Properties of Metals (Pergamon, New York, 1978).

${ }^{9} \mathrm{http}: / /$ www.flapw.de.

${ }^{10}$ E. Fawcett, Rev. Mod. Phys. 60, 209 (1988).

${ }^{11}$ J. Inglesfield, J. Phys. Chem. 14, 3795 (1981).

${ }^{12}$ D. Wortmann, H. Ishida, and S. Blügel, Phys. Rev. B 66, 075113 (2002).

${ }^{13}$ D. Wortmann, H. Ishida, and S. Blügel, Phys. Rev. B 65, 165103 (2002).

${ }^{14}$ R. Landauer, IBM J. Res. Dev. 1, 223 (1957). 
${ }^{15}$ J. A. Stroscio, D. T. Pierce, A. Davies, R. J. Celotta, and M. Weinert, Phys. Rev. Lett. 75, 2960 (1995).

${ }^{16}$ J. Bardeen, Phys. Rev. Lett. 6, 57 (1960).

${ }^{17}$ D. Wortmann, H. Ishida, and S. Blügel, Phys. Rev. B 72, 235113 (2005)

${ }^{18}$ S. Ohnishi, A. J. Freeman, and M. Weinert, Phys. Rev. B 28, 6741 (1983).

${ }^{19}$ T. Asada, G. Bihlmayer, S. Handschuh, S. Heinze, P. Kurz, and S. Blügel, J. Phys.: Condens. Matter 11, 9347 (1999).

${ }^{20}$ H. Zabel, J. Phys.: Condens. Matter 11, 9303 (1999).

${ }^{21}$ E. Fawcett, Rev. Mod. Phys. 60, 209 (1988).

${ }^{22}$ S. Blügel, D. Pescia, and P. H. Dederichs, Phys. Rev. B 39,
R1392 (1989).

${ }^{23}$ C. L. Fu and A. J. Freeman, Phys. Rev. B 33, 1755 (1986).

${ }^{24}$ O. Y. Kolesnychenko, R. de Kort, M. I. Katsnelson, A. I. Lichtenstein, and H. van Kempen, Nature (London) 415, 507 (2003).

${ }^{25}$ P. Mavropoulos, N. Papanikolaou, and P. H. Dederichs, Phys. Rev. Lett. 85, 1088 (2000).

${ }^{26}$ W. H. Butler, X.-G. Zhang, T. C. Schulthess, and J. M. MacLaren, Phys. Rev. B 63, 092402 (2001).

${ }^{27}$ I. Mazin, Europhys. Lett. 55, 404 (2001).

${ }^{28}$ J. Tersoff and D. R. Hamann, Phys. Rev. Lett. 50, 1998 (1983).

${ }^{29}$ D. Wortmann, S. Heinze, P. Kurz, G. Bihlmayer, and S. Blügel, Phys. Rev. Lett. 86, 4132 (2001). 